Nicholas M. Allen, $\mathrm{MB}, \mathrm{BCh}, \mathrm{MD}$

Yael Hacohen, MD

Jacqueline Palace, MD

David Beeson, PhD

Angela Vincent, PhD

Heinz Jungbluth, MD, $\mathrm{PhD}$

\section{SALBUTAMOL-RESPONSIVE FETAL ACETYLCHOLINE RECEPTOR INACTIVATION SYNDROME}

Transient neonatal myasthenia gravis (TNMG) is a rare and usually self-limiting condition due to maternal acetylcholine receptor (AChR) antibodies in neonates born to mothers with myasthenia gravis (MG). However, a skeletal myopathy with consistent and recognizable features (fetal acetylcholine receptor inactivation syndrome [FARIS]) was recently reported in some patients. ${ }^{1,2}$ FARIS occurs in association with clinically manifest maternal MG or asymptomatically elevated maternal AChR antibodies directed against the fetal AChR $\gamma$ subunit. While in utero antibody exposure is considered the main pathogenic mechanism, the timing and intensity of maternal MG treatment plays an important role in determining fetal and postnatal outcome.

The clinical spectrum of FARIS is wide, ranging from lethal arthrogryposis multiplex congenita (AMC) to mild, mainly facial myopathic manifestations persisting into adulthood. ${ }^{1,3}$ While the symptoms of typical TNMG often respond to acetylcholinesterase inhibition or removal of causative antibodies, FARIS does not respond to the same treatments, likely reflecting antibodymediated structural endplate alterations of antenatal onset, given that the $\gamma$-subunit is required for the assembly of pre-patterned AChR clusters and, ultimately, neuromuscular synaptogenesis.

We report a child with FARIS showing dramatic symptom improvement following therapy with salbutamol (albuterol), a short-acting $\beta 2$-adrenergic receptor agonist previously used effectively in specific genetic forms of congenital myasthenic syndrome (CMS).

Level of evidence. This study provides Class IV evidence that salbutamol improves myasthenia-related signs in children with FARIS. This is a single observational study without controls.

Case report. The proband (patient 2.2 in reference 1) is the second child of a previously reported family with FARIS. ${ }^{1,5}$ His mother was diagnosed with MG in her first pregnancy. There was an older sibling with lethal
Clinical/Scientific Notes

AMC, and a younger brother with much milder symptoms, probably reflecting different treatment intensities of maternal MG over consecutive pregnancies. He was intubated at birth and ventilated for 3 weeks. He was profoundly hypotonic with a weak fatigable suck and required nasogastric tube feeding, followed by temporary gastrostomy for 9 months. Early examinations revealed motor delay and marked axial, facial, and bulbar weakness. He received pyridostigmine from birth until his second year, with little benefit beyond 2-3 months. Despite appropriate developmental milestones at 20 months, he had persistent facial diplegia with severe oromotor language difficulties, a weak voice, drooling, and substantially reduced stamina.

Examination at almost 5 years demonstrated severe facial muscle weakness, with an open mouthed expression (figure, A), a high arched palate, bilateral ptosis, and limited upgaze. His voice was dysarthric, dysphonic, and weak (sentences only understood by his mother). Antigravity movements in neck flexion were limited (Medical Research Council [MRC] grade 3-15) but power was normal in all other muscles. There was no added fatigability. He was treated with oral salbutamol (albuterol) at an initial dose of $1 \mathrm{mg} 3$ times daily. Within 48 hours, a dramatic improvement in facial expression and definition including ptosis (figure, B), quality of voice and speech (now understood by most adults), general stamina (significantly reduced rest periods), and drooling frequency was noted. Salbutamol was increased to $2 \mathrm{mg} 3$ times daily, resulting in further improvement (figure, C), confirmed on examination at 4 years 9 months. Neck flexion was MRC 3+/5. Salbutamol benefits were sustained and treatment was well-tolerated.

Discussion. FARIS ${ }^{1,2}$ is a recently recognized, early-onset myopathy associated with maternal antibodies against the fetal AChR $\gamma$ subunit that is crucial for the normal development and functioning of the embryonic neuromuscular junction (NMJ), a notion supported by the marked abnormalities seen in patients with Escobar syndrome due to recessive mutations in the CHRNG gene ${ }^{6}$ causing fetal AChR $\gamma$ subunit disruption. Absence of myasthenic features on neurophysiologic assessments ${ }^{1}$ 

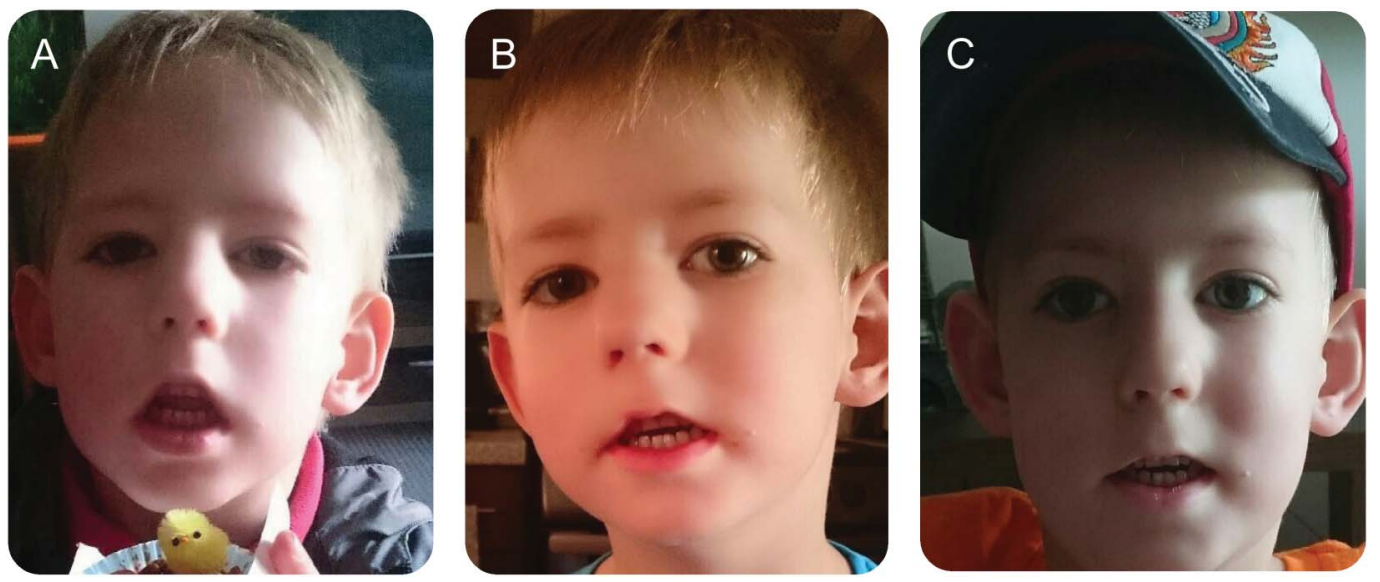

Patient with fetal acetylcholine receptor inactivation syndrome at 4 years 9 months of age. Facial features (A) before, (B) 24 hours after commencement of treatment with salbutamol $1 \mathrm{mg} 3$ times daily, and (C) 24 hours after increase of salbutamol treatment to $2 \mathrm{mg} 3$ times daily.

\section{Comment:}

\section{Salbutamol-}

\section{A means to an endplate}

The fetal acetylcholine receptor (AChR) inactivation syndrome (FARIS) was first described in the last decade, resulting from maternal AChR antibodies directed against the fetal $\gamma$ subunit of the receptor. The phenotype is myopathic, ranging from a lethal arthrogryposis multiplex congenita to mild weakness. Unlike transient neonatal myasthenia gravis $(\mathrm{MG})$, wherein the hypotonia and weakness usually resolve within a few months, and if anticholinesterase therapy is needed, it can usually be withdrawn after $4-6$ weeks, ${ }^{1}$ FARIS is persistent and typically has poor responsiveness to agents such as pyridostigmine and immunotherapy.

In this single case report, ${ }^{2}$ investigators from Oxford, an expert group in both immune-mediated and congenital disorders of neuromuscular transmission, describe the dramatic response of a 5-year-old boy with FARIS to oral salbutamol, better known as albuterol in North America. Although pyridostigmine did not improve facial and bulbar symptoms, salbutamol doses up to $6 \mathrm{mg}$ total a day improved facial expression and definition, speech production, and stamina. In the photographs provided, ptosis also appears improved.

$\beta 2$-Adrenergic agonists such as salbutamol have been used successfully in some forms of congenital myasthenic syndrome, primarily those that are postsynaptic. Less-specific adrenergic agonists such as ephedrine as well as $\beta 2$ agonists have also been used in MG with some success. A double-blind, placebo-controlled crossover study of oral terbutaline $2.5 \mathrm{mg} 3$ times daily demonstrated efficacy in about twothirds of patients with MG. ${ }^{3}$ Terbutaline was well-tolerated with only mild tremors reported. The ability of adrenergic agents to promote quantal release and enhance muscle twitch tension may underlie their favorable effects on both disorders of neuromuscular transmission and the endplate myopathy proposed to cause FARIS. The dramatic response illustrated in the report of this young boy offers guidance for both pediatric and neuromuscular neurologists who encounter such cases.

1. Muppidi S, Wolfe GI, Barohn RJ. Diseases of the neuromuscular junction. In: Swaiman KF, Ashwal S, Ferriero DM, Schor NF, eds. Pediatric Neurology: Principles and Practice, 5th ed. Philadelphia: Elsevier Saunders; 2012:1549-1569.

2. Allen NM, Hacohen Y, Palace J, Beeson D, Vincent A, Jungbluth H. Salbutamol-responsive fetal acetylcholine receptor inactivation syndrome. Neurology 2016;86:692-694.

3. Soliven B, Rezania K, Gundogdu B, Harding-Clay B, Oger J, Arnason BGW. Terbutaline in myasthenia gravis: a pilot study. J Neurol Sci 2009;277:150-154.

Gil I. Wolfe, MD Nicholas J. Silvestri, MD

From the Department of Neurology, University at Buffalo Jacobs School of Medicine and Biomedical Sciences, State University of New York, Buffalo.

Study funding: No targeted funding reported.

Disclosure: Drs. Wolfe and Silvestri have received research support from Alexion. Dr. Wolfe serves on advisory boards for Baxalta, Grifols, CSL Behring, and Syntimmune. Dr. Silvestri serves on an advisory board for Alexion. Go to Neurology.org for full disclosures. and lack of response to acetylcholinesterase inhibitors in the chronic stages suggested that FARIS represented an ingrained endplate myopathy rather than an ongoing defect of neuromuscular transmission. ${ }^{1}$

Salbutamol, a $\beta 2$-adrenergic agonist commenced in our patient considering its proven efficacy in various neuromuscular disorders and lack of response to other treatments, resulted in dramatic and sustained improvement. Salbutamol is highly effective in genetic CMS due to mutations in several genes, most notably DOK7 and COLQ, and has been demonstrated to improve AChR cluster assembly and NMJ architecture in a mouse model of anti-MuSK MG.?

Our observations suggest that salbutamol is an effective therapy for a potentially severe condition for which there is currently no treatment. ${ }^{1}$ FARIS may provide a suitable model to study mechanisms of salbutamol actions also relevant to other neuromuscular conditions with disturbed NMJ architecture or function.

From Evelina's Children Hospital (N.M.A., H.J.), Guy's \& St. Thomas' Hospital NHS Foundation Trust, London; University of Oxford (Y.H., D.B., A.V.); John Radcliffe Hospital (J.P.), Oxford; and King's College (H.J.), London, UK.

Author contributions: N.M. Allen obtained the data and wrote the first draft of the manuscript. Y. Hacohen obtained the original data and revised the manuscript for critical content. J. Palace, D. Beeson, and A. Vincent revised the manuscript for critical content. H. Jungbluth initiated and supervised the project and revised the manuscript for critical content.

Acknowledgment: The authors thank the patient's parents for their participation and consent to publication of clinical photographs; Dr. Leslie Jacobson for antibody testing; and Dr. Darryl de Vivo, New York, NY, for stimulating discussions.

Study funding: No targeted funding reported.

Disclosure: N.M. Allen, Y. Hacohen, J. Palace, and D. Beeson report no disclosures relevant to the manuscript. A. Vincent receives royalties for antibody assays not relevant to this study. H. Jungbluth reports no 
disclosures relevant to the manuscript. Go to Neurology.org for full disclosures.

Received July 24, 2015. Accepted in final form October 7, 2015.

Correspondence to Dr. Jungbluth: heinz.jungbluth@gstt.nhs.uk

(C) 2016 American Academy of Neurology

1. Hacohen Y, Jacobson LW, Byrne S, et al. Fetal acetylcholine receptor inactivation syndrome: a myopathy due to maternal antibodies. Neurol Neuroimmunol Neuroinflamm 2014;2:e57.

2. Oskoui M, Jacobson L, Chung WK, et al. Fetal acetylcholine receptor inactivation syndrome and maternal myasthenia gravis. Neurology 2008;71:2010-2012.

3. Reimann J, Jacobson L, Vincent A, Kornblum C. Endplate destruction due to maternal antibodies in arthrogryposis multiplex congenita. Neurology 2009;73: 1806-1808.

4. Liu Y, Padgett D, Takahashi M, et al. Essential roles of the acetylcholine receptor gamma-subunit in neuromuscular synaptic patterning. Development 2008;135: 1957-1967.

5. Chieza JT, Fleming I, Parry N, Skelton VA. Maternal myasthenia gravis complicated by fetal arthrogryposis multiplex congenita. Int J Obstet Anesth 2011;20:79-82.

6. Hoffmann K, Muller JS, Stricker S, et al. Escobar syndrome is a prenatal myasthenia caused by disruption of the acetylcholine receptor fetal gamma subunit. Am J Hum Genet 2006;79:303-312.

7. Ghazanfari N, Morsch M, Tse N, Reddel SW, Phillips WD. Effects of the 32 -adrenoceptor agonist, albuterol, in a mouse model of anti-MuSK myasthenia gravis. PLoS One 2014;9:e87840. 